\title{
Legionella pneumophila exhibits plasminogen activator activity
}

\author{
Correspondence \\ Jozef Anné \\ Jozef.Anne@rega.kuleuven.be
}

Received 29 May 2007

Revised 13 August 2007

Accepted 14 August 2007

\author{
Leen Vranckx, Emmy De Buck, Jozef Anné and Elke Lammertyn
}

Laboratory for Bacteriology, Rega Institute for Medical Research, Katholieke Universiteit Leuven, Minderbroedersstraat 10, B-3000 Leuven, Belgium

\begin{abstract}
Based on their localization at the boundary of the bacterial cell and its environment, outermembrane proteins (Omps) are important determinants for interaction of bacteria with their host cell. Therefore, they can be considered as important determinants for virulence. Looking for Legionella pneumophila Omps potentially involved in virulence, we identified a gene encoding a homologue of the plasminogen activator $(\mathrm{Pla})$ of Yersinia pestis. Pla belongs to the class of omptins, a family of surface proteases/adhesins that exhibit different virulence-associated functions. In this report we describe the cloning and identification of the plasminogen activator homologue Lpa of $L$. pneumophila and demonstrate its outer-membrane localization.

Transcriptional analysis of the Lpa region revealed expression of the gene in both exponential and stationary growth phase and showed that transcription of the Ipa gene is directed by its own promoter. We also show, to our knowledge for the first time, that $L$. pneumophila has the capacity to convert plasminogen into plasmin by the action of the outer-membrane Lpa protein.
\end{abstract}

\section{INTRODUCTION}

Legionella pneumophila is a Gram-negative, facultative intracellular human pathogen found worldwide in freshwater environments, where it replicates in various protozoa. Intracellular growth enhances the environmental survival capability and the virulence of the bacteria. People typically become infected with $L$. pneumophila upon inhalation of contaminated aerosols generated by manmade devices such as whirlpools, cooling towers and airconditioning systems. If the host immune response is impaired, adhesion to and infection of alveolar macrophages can lead to Legionnaires' disease, a life-threatening pneumonia. Since its discovery in 1976, but particularly in the past 10 years, our understanding of the pathogenesis of L. pneumophila has grown tremendously. Both the unravelling of the biphasic life cycle of Legionella, in which two clearly distinct phenotypes can be differentiated (Molofsky \& Swanson, 2004; Molmeret et al., 2005; Brüggemann et al., 2006; Hilbi et al., 2007), together with the identification of a great number of virulence factors, form the basis of the progress made (Albert-Weissenberger et al., 2007). In recent years the number of reported cases of legionellosis has risen dramatically, resulting in increased attention to control measurements to prevent infections by this pathogen and to the identification and mechanism of action of additional virulence factors. A variety of L. pneumophila surface components and secreted

Abbreviations: $\alpha_{2} \mathrm{AP}, \alpha_{2}$-antiplasmin; Lpa, Legionella plasminogen activator; MOMP, major outer-membrane protein; Omps, outer-membrane proteins; Pla, plasminogen activator; Plg, plasminogen. proteins have been shown to be implicated in pathogenesis. In several bacterial pathogens, functional characterization of outer-membrane proteins (Omps) has revealed diverse roles for these molecules, including iron uptake, antimicrobial peptide and multi-drug resistance, bile resistance, pore formation, adhesion, invasion, bacteriophage adsorption and serum resistance. Although significant advances have been made regarding the unravelling of the structure and function of Omps, the number of Omps that have been characterized represents only a small portion of the total number of Omps revealed by bacterial genome sequences (Lin et al., 2002).

In the search for L. pneumophila genes encoding putative Omps, and more precisely Omps that might be involved in bacterial virulence, we identified a gene encoding a homologue of the plasminogen activator protein (Pla) of Yersinia pestis. The latter protein belongs to the family of the omptins, which is a class of surface proteases/adhesins found in the outer membrane of enterobacterial pathogens.

Although the omptin homologues show high sequence identity and a conserved $\beta$-barrel, they are multifunctional and exhibit different virulence-associated functions. Pla of $Y$. pestis contributes to the virulence of this pathogen by several mechanisms. The protein is involved in uncontrolled plasmin activity by efficient conversion of human proenzyme plasminogen (Plg) to plasmin, inactivation of the plasmin inhibitor $\alpha_{2}$-antiplasmin $\left(\alpha_{2}\right.$ AP), and binding to laminin, which localizes the plasmin activity onto basement membranes. These properties are important for the bacterial invasiveness in plague (Sodeinde et al., 1992) 
and enhance migration of $Y$. pestis through tissue barriers (Kukkonen \& Korhonen, 2004). PgtE, the homologue of $\mathrm{Pla}$ in Salmonella enterica, is significantly less efficient in plasminogen activation than Pla (Kukkonen et al., 2004), but inactivates the plasmin inhibitor $\alpha_{2} \mathrm{AP}$ more efficiently (Lähteenmäki et al., 2005). This discrepancy may reflect differences in the mechanisms of infection exerted by these two pathogens. The generation of plasmin is indispensable for the predominantly extracellular $Y$. pestis to proteolyse tissues in order to disseminate all over the human body. Since spread of $S$. enterica occurs predominantly by means of macrophages, Lähteenmäki et al. (2005) hypothesize that PgtE-mediated inactivation of $\alpha_{2} \mathrm{AP}$ serves to protect the plasmin generated by both the bacteria and the macrophages. Based on these results obtained for other bacterial pathogens, we hypothesized that the L. pneumophila omptin homologue could play an important role in the virulence of this pathogen as well.

In this study, we cloned the gene encoding the $L$. pneumophila plasminogen activator homologue and demonstrated, to our knowledge for the first time, that $L$. pneumophila possesses a surface protease that exhibits plasminogen activator activity.

\section{METHODS}

Strains, plasmids and cultivation of bacteria and cells. Strains used in this study are listed in Table 1. Legionella strains were grown at $37{ }^{\circ} \mathrm{C}$ on buffered charcoal yeast extract (BCYE) agar plates or in buffered yeast extract broth (BYE) supplemented with $\alpha$-ketoglutarate, L-cysteine and ferric pyrophosphate (Edelstein, 1981). When necessary, kanamycin $\left(10 \mu \mathrm{g} \mathrm{ml}^{-1}\right)$ or chloramphenicol (5$12 \mu \mathrm{g} \mathrm{ml}^{-1}$ ) was added. Escherichia coli TG1 (Sambrook et al., 1989) was used as host for cloning purposes and E. coli BL21* and E. coli BL21(DE3)pLys were used for the expression of recombinant proteins (Studier \& Moffatt, 1986). Cultures were grown in LuriaBertani medium (Miller, 1972) and if necessary supplemented with ampicillin $\left(50 \mu \mathrm{g} \mathrm{ml}^{-1}\right)$ or chloramphenicol $\left(50 \mu \mathrm{g} \mathrm{ml}^{-1}\right)$. Acanthamoeba castellanii (ATCC 30234) was cultured in PYG712 medium at room temperature.

DNA manipulations and cloning strategies. All DNA manipulations used in this work were performed using standard techniques unless specified otherwise. The plasmids and primers used in this study are given in Tables 1 and 2, respectively. Standard PCR reactions were carried out using SuperTaq (HT Biotechnology).

The L. pneumophila lpa gene was isolated by PCR using the primers LpaF and LpaR, from chromosomal DNA (100 ng) as template. The resulting $0.92 \mathrm{~kb}$ PCR fragment was cloned into pGEM-T Easy (Promega). Subsequently, the lpa gene was cloned downstream of the IPTG-inducible tac promoter in $\mathrm{pMMBN}$. The $\mathrm{pMMBN}$ vector is a derivative of pMMB207 (Morales et al., 1991) with an extra NdeI site introduced at the start codon of the lac $Z \alpha$ gene and with part of the mobA gene deleted by AgeI digestion and religation. In this way, the plasmid pMMBNlpa was obtained.

Using the primers PromlpaF and LpaR the lpa gene with its promoter region was amplified by PCR from chromosomal DNA (100 ng) as template. The resulting $1.2 \mathrm{~kb}$ PCR fragment was cloned into pGem$\mathrm{T}$ Easy (Promega) and subsequently as a SacI-SacII restriction fragment cloned into $\mathrm{pBCKS}(+)$. In this way, the plasmid pBCpromlpa containing the gene together with its putative promoter region was obtained.

Table 1. Strains and plasmids

\begin{tabular}{|c|c|c|}
\hline Name & Relevant characteristics ${ }^{\star}$ & Reference or source \\
\hline \multicolumn{3}{|l|}{ Strains } \\
\hline \multicolumn{3}{|l|}{ L. pneumophila } \\
\hline Philadelphia & L. pneumophila serogroup 1 & ATCC 33152 \\
\hline Philadelphia $\Delta l p a$ & Philadelphia $l_{p a}:: \mathrm{Km}$ & This study \\
\hline Corby & L. pneumophila serogroup 1 & Lück et al. (2001) \\
\hline Corby LPS mutant & Spontaneous LPS mutant from L. pneumophila sg 1 strain Corby & Lück et al. (2001) \\
\hline \multicolumn{3}{|c|}{ 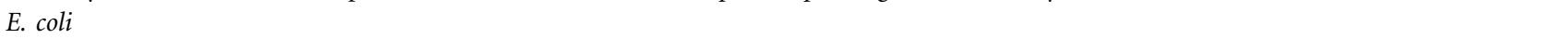 } \\
\hline TG1 & Host for cloning purposes & Studier \& Moffatt (1986) \\
\hline $\mathrm{BL} 21^{*}$ & Host for overexpression & Novagen \\
\hline BL21(DE3)pLys & Host for overexpression & Novagen \\
\hline A. castellanii & & ATCC 30234 \\
\hline \multicolumn{3}{|l|}{ Plasmids } \\
\hline pGEM-T Easy & Used for cloning of PCR-amplified DNA fragments, MCS, $\mathrm{Ap}^{\mathrm{r}}$, lac $Z \alpha$ & Promega \\
\hline pGEMlpa & pGEM-T Easy derivative containing the $l p a$ fragment & This study \\
\hline pGEMpromlpa & pGEM-T Easy derivative containing the promlpa fragment & This study \\
\hline pGEMlpaHis & pGEM-T Easy derivative containing the $(\mathrm{His})_{10} l p a$ fragment & This study \\
\hline pMMB207 & E. coli-Legionella shuttle vector, $l a c Z \alpha, \mathrm{Cm}^{\mathrm{r}}$ & Morales et al. (1991) \\
\hline pMMBN & pMMB207 derivative with an extra NdeI site and part of the mobA gene deleted & De Buck et al. (2007) \\
\hline pMMBNlpa & pMMBN derivative containing the $l p a$ fragment of pGEMlpa & This study \\
\hline $\operatorname{pBCKS}(+)$ & $\mathrm{MCS}, \mathrm{Cm}^{\mathrm{r}}, l a c Z \alpha$ & Stratagene \\
\hline pBCpromlpa & pBCKS $(+)$ derivative containing the promlpa fragment of pGEMpromlpa & This study \\
\hline pETlpaHis & pET3a derivative containing the (His) ${ }_{10} l p a$ fragment of pGEMlpaHis & This study \\
\hline
\end{tabular}

${ }^{\star} \mathrm{Ap}^{\mathrm{r}}$, ampicillin resistance; $\mathrm{Cm}^{\mathrm{r}}$, chloramphenicol resistance; MCS, multiple cloning site. 
Table 2. Primers

Restriction endonuclease cleavage sites are underlined. The (His) ${ }_{10}$-encoding sequence is in italic.

\begin{tabular}{|lll|}
\hline Name & \multicolumn{1}{|c|}{ Sequence $\left(5^{\prime}-3^{\prime}\right)$} & \multicolumn{1}{c|}{ Feature } \\
\hline LpaF & tacatatgaaaaataaaatagttttgttggc & NdeI \\
PromlpaF & ggttttgccctattgtcc & \\
LpaR & tagaattcttaaaaaagatactgtaaacc & EcoRI \\
LpaFHis & tacatatgcaccatcaccatcatcatcaccatcatcacgcggattacgaatttaatgg & NdeI, (His) $10^{\text {-tag }}$ \\
\hline
\end{tabular}

For the construction of an $L$. pneumophila Philadelphia mutant lacking the lpa gene, the chromosomal lpa gene was substituted by a kanamycin-resistance gene by means of double homologous recombination as described for other L. pneumophila genes (De Buck et al., 2005). To make sure that the observed effects after deletion of the lpa gene could be specifically ascribed to the deletion of the lpa gene, we constructed a complemented L. pneumophila lpa deletion strain by introducing pMMBNlpa into the mutant strain. A second complemented strain with lpa under transcriptional control of its own promoter was constructed by introducing $\mathrm{pBCpromlpa}$ into the mutant strain.

To construct pETlpaHis, the lpa gene was amplified by PCR using LpaFHis and LpaR. The forward primer was designed to add ten histidines at the $\mathrm{N}$-terminal end of the mature protein. After cloning into pGEM-T Easy, the fragment was digested with NdeI and ligated into pET3a.

RNA isolation and reverse transcriptase (RT)-PCR. Total RNA from exponentially growing and stationary-phase L. pneumophila cells and from L. pneumophila intracellularly grown in A. castellanii was isolated using the RNeasy mini kit (Qiagen) as described previously (De Buck et al., 2004). Samples used for RT-PCR were additionally treated with DNase I (Qiagen) (final concentration 0.5 units $\mu^{-1}$ ) to remove any residual chromosomal DNA. RT-PCR was performed with 100 ng of total RNA using the Access RT-PCR System (Promega). To investigate the presence of an operon structure encompassing the lpa gene, the primers PromlpaF and LpaR were used.

Cell fractionation. For the preparation of protein fractions containing either extracellular proteins, total cell proteins, soluble, inner- or outer-membrane proteins, a $100 \mathrm{ml}$ culture was grown overnight starting from a $1 \mathrm{ml}$ overnight-grown preculture. The culture was centrifuged $(5000 \mathrm{~g}, 15 \mathrm{~min})$ and the supernatant obtained was filter-sterilized using a $0.2 \mu \mathrm{m}$ filter. Proteins present in the supernatant were concentrated by TCA precipitation $(20 \%$ TCA) for $1 \mathrm{~h}$ on ice. After centrifugation $(10000 \mathrm{~g}, 15 \mathrm{~min})$ the extracellular protein fraction was washed with acetone and dried. The remaining cell pellet was resuspended in $15 \mathrm{ml}$ lysis buffer $(10 \mathrm{mM}$ Tris, $10 \mathrm{mM}$ EDTA, $\mathrm{pH}$ 7.5) and subsequently lysed in a French pressure cell. To obtain the total cell protein fraction, the cell debris was removed by centrifugation $(20 \mathrm{~min}, 12000 \mathrm{~g}$ ). Membrane proteins and the soluble protein fraction were obtained following centrifugation of the cell lysate for $2 \mathrm{~h}$ at $100000 \mathrm{~g}$. The soluble protein fraction is located in the supernatant, whereas the membranes with the membrane proteins are situated in the sediment. To set the inner-membrane proteins free from the membranes, the sediment was resuspended in $1.5 \mathrm{ml} 10 \mathrm{mM}$ Tris/ $\mathrm{HCl}$ ( $\mathrm{pH} 7.5$ ) containing $1.5 \%$ Sarkosyl and incubated for $30 \mathrm{~min}$ at room temperature, and subsequently recentrifuged for $2 \mathrm{~h}$ at $100000 \mathrm{~g}$. The supernatant, containing the inner-membrane proteins, was collected. The pellet, containing the outer-membrane proteins, was resuspended in $500 \mu \mathrm{l}$ $10 \mathrm{mM}$ Tris/ $\mathrm{HCl}(\mathrm{pH} 7.5)$ and $10 \mathrm{mM}$ EDTA, containing $1 \%$ Triton $\mathrm{X}-100$.
SDS-PAGE and Western blotting. (His) ${ }_{10}$-Lpa was isolated from the cytoplasm of $E$. coli BL21(DE3)pLys/pETLpaHis under denaturing conditions as described in Qia Expressionist (Qiagen). To obtain extra pure protein for immunization purposes, purified fractions were additionally separated by SDS-PAGE and subsequently the Lpa protein was electro-eluted from the gels. After dialysis of the purified fractions against PBS, $150 \mu \mathrm{g}$ of the (His) $10^{-L}$ pa protein was used for immunization of a Dutch White rabbit, in order to obtain Lpaspecific antibodies.

Expression of Lpa was monitored by Western blotting (12.5\% SDSPAGE) and immunodetection with Lpa-specific antibodies and in a second step with alkaline-phosphatase-conjugated anti-rabbit antibodies (Sigma). Visualization of the proteins was done using the chromogenic substrate solution NBT/BCIP (Roche Diagnostics).

As a control for the protein fractionation, detection of L. pneumophila DnaK, a cytoplasmic protein, LepB (signal peptidase), an innermembrane protein, and MOMP (major outer-membrane protein), an outer-membrane protein, was performed with antibodies against $E$. coli DnaK, LepB and MOMP, respectively.

Plasminogen activation tests. Measurement of plasminogen activation was performed as described previously (Kukkonen et al., 2001), by incubating $8 \times 10^{7}$ bacteria, $4 \mu \mathrm{g}$ human Glu-Plg (American Diagnostica) and the chromogenic plasmin substrate S-2251 (0.45 mM Val-Leu-Lys- $p$-nitroaniline dihydrochloride, Chromogenix) in a total volume of $200 \mu \mathrm{l}$ at $37{ }^{\circ} \mathrm{C}$. Plasmin activity was measured in the Infinite 200 microplate reader (Tecan) at $405 \mathrm{~nm}$ as the breakdown of the chromogenic substrate as a function of time. Activity of inner- and outer-membrane protein fractions was measured by the same test as described above, but instead of bacteria, $20 \mu \mathrm{l}$ of these fractions (see preparation above) was used in the samples.

Amoebae plate test. Growth of L. pneumophila in the presence of $A$. castellanii was analysed as described by Albers et al. (2005). Briefly, $1.5 \mathrm{ml} \mathrm{A}$. castellanii $\left(2.6 \times 10^{6} \mathrm{ml}^{-1}\right)$ was spread on BCYE agar plates, allowed to dry for $1-2 \mathrm{~h}$ in a laminar-flow hood and left overnight at room temperature. Stationary-phase bacterial cultures were adjusted to an identical $\mathrm{OD}_{600}$ and series of tenfold dilutions in BYE were prepared; $3 \mu \mathrm{l}$ of stationary culture and of each dilution was spotted onto the BCYE agar plate and the plates were incubated for 5 days at $37^{\circ} \mathrm{C}$.

\section{RESULTS AND DISCUSSION}

\section{Cloning and analysis of the Ipa gene of $L$. pneumophila}

In order to identify novel virulence factors of $L$. pneumophila, the genome sequence of $L$. pneumophila Philadelphia was screened for the presence of genes 
encoding proteins that are associated with the bacterial outer membrane using the Psortb algorithm. One of the genes resulting from this search $(\operatorname{lpg} 2387)$ was predicted to encode a plasminogen activator homologue. For this reason we named the gene lpa (Legionella plasminogen activator). Homologues in the Paris and Lens strain are lpp2452 and lpl2311, respectively. The gene was cloned by PCR from L. pneumophila Philadelphia genomic DNA and the DNA sequence present in the genome bank could be confirmed. The cloned fragment encodes a protein of 308 amino acids with a calculated molecular mass of $35.3 \mathrm{kDa}$ and a predicted $\mathrm{pI}$ of 6.0. Analysis of the predicted protein sequence with SignalP confirmed the presence of a typical signal peptide at the $\mathrm{N}$ terminus, with the most likely cleavage site between the amino acids at positions 23 and 24 .

The protein sequence of L. pneumophila Lpa was compared to the omptin sequences of other Gram-negative human pathogens: E. coli, $Y$. pestis and S. enterica serovar Typhimurium (Fig. 1). Overall identity of Lpa with these proteins is respectively 39,49 and $47 \%$. The structure of the best-studied member of the omptin family, OmpT, elucidated by Vandeputte-Rutten et al. (2001), appears to consist of a $\beta$-barrel with ten antiparallel $\beta$-strands connected by four short periplasmic turns and five surface-exposed loops. The extracellular loops of OmpT are indicated in Fig. 1.

Although the omptins are structurally very similar and the catalytically important residues (Vandeputte-Rutten et al., 2001; Kramer et al., 2000, 2001) are conserved (Fig. 1), they have clearly different substrates (Kukkonen \& Korhonen, 2004). It is assumed that this substrate specificity is dependent on slight differences in the amino acid sequence in the regions important for substrate recognition in the extracellular loops of the protein. There are three important structural features to convert the substrate specificity of OmpT into that of Pla and as a result change it into a protein with enhanced plasminogen activator activity (Kukkonen et al., 2001). The three key elements are (i) shortening of L3 by four residues to the size of the Pla L3, (ii) deletion of two amino acids in L4, i.e. $\mathrm{Asp}^{213}$ and Pro $^{214}$, and (iii) substitution of residue Lys ${ }^{216}$ by Arg in L4. These typical plasminogen activator-related structural features are also present in the L. pneumophila protein, leading to the hypothesis that this protein exhibits plasminogen activator activity.

\section{Transcriptional analysis of the L. pneumophila Ipa gene}

On the L. pneumophila Philadelphia chromosome the lpa gene is situated between a putative ORF with unknown function (lpg2386) and a gene encoding an amino acid permease (lpg2388) (Fig. 2a). The latter is oriented in the same direction as $l p a$ with an intergenic distance of $130 \mathrm{bp}$. RT-PCR experiments on total L. pneumophila RNA were performed to see whether the lpa gene is effectively expressed under the experimental conditions used and to determine if it is cotranscribed with the upstream ORF. RT-PCR with lpa-specific primers clearly showed the presence of a $0.92 \mathrm{~kb}$ transcript in the case of $L$. pneumophila grown to exponential and stationary phase, confirming lpa expression in both phases (Fig. 2b). The lpa gene is also expressed in intracellularly grown bacteria (Fig. 2b). Furthermore, no transcript that encompasses the intergenic region could be obtained, indicating that transcription of the lpa gene is directed by its own promoter. RT-PCR on RNase-treated samples was negative, confirming a specific, DNA-independent amplification.

\section{Cellular localization of Lpa}

Based on homology with other omptin proteins and on an in silico prediction, we assumed that the L. pneumophila Lpa protein is located in the outer membrane of the bacterial cells. In order to confirm this localization, different protein fractions were studied for the presence of Lpa. We first started with the analysis of total cell lysates of $L$. pneumophila. However, after SDS-PAGE of total cell proteins and detection with Lpa-specific antibodies, Lpa could only be detected in tiny amounts (Fig. 3). When the same analysis was performed with total cell proteins of $L$. pneumophila/pMMBNlpa, overexpressing the lpa gene upon IPTG induction, a clear band at a molecular mass of $33 \mathrm{kDa}$, corresponding to the predicted molecular mass of $32.8 \mathrm{kDa}$ of the mature form of Lpa, could be observed (Fig. 3).

Separation of the different cellular fractions by SDS-PAGE, followed by immunodetection with Lpa-specific antibodies, revealed no immunoreactive protein band in the extracellular, soluble and inner-membrane protein fraction, while a clear band was observed in the outermembrane protein fraction (Fig. 3). As a control for a correct cellular fractionation, detection of $L$. pneumophila DnaK, a cytoplasmic protein, LepB (signal peptidase), an inner-membrane protein, and MOMP, an outer-membrane protein, was performed. The control proteins were found to be present in the expected fractions (results not shown). Based on these experiments we could conclude that Lpa is an outer-membrane protein.

\section{Functional analysis of Lpa}

We studied the capacity of Lpa to convert plasminogen to plasmin with plasminogen activator tests on cells and on inner- and outer-membrane protein fractions. As stated above, the capacity to convert plasminogen into plasmin is very different for each of the characterized omptins. For example, the Pla protein of $Y$. pestis shows a more efficient activation of Plg than OmpT of E. coli and PgtE of $S$. enterica (Kukkonen et al., 2004). In order to assess the plasminogen activation ability of the Lpa protein, its activity was monitored as a function of time for $L$. pneumophila cells and L. pneumophila/pMMBNlpa cells with and without induction with IPTG. For a similar experiment in E. coli, the OmpT-deficient E. coli BL21* was 


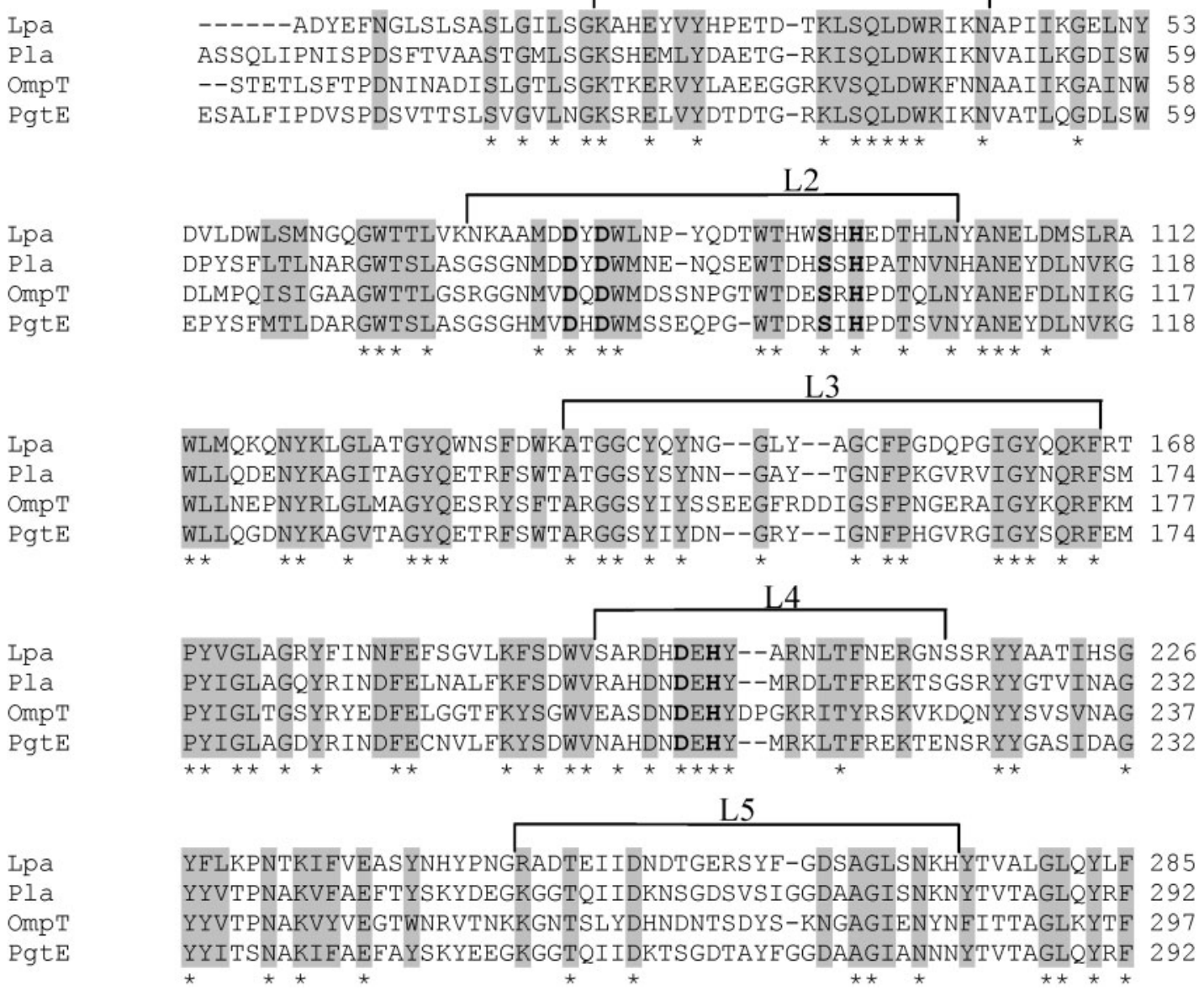

Fig. 1. Alignment of the amino acid sequence of the mature part of L. pneumophila Lpa with known omptins of other Gramnegative pathogens: Pla of $Y$. pestis, OmpT of E. coli and PgtE of S. enterica. Sequences were aligned with CLUSTAL W. Conserved residues are shaded and identical residues are marked by an asterisk. Catalytically important residues are indicated in bold and extracellular loops determined for OmpT are indicated by lines above the sequences (Vandeputte-Rutten et al., 2001).

used as host strain in order to minimize interference of residual chromosomally encoded plasminogen activator activity. Results of both tests are given in Fig. 4.

For L. pneumophila, no activation of plasminogen could be detected under non-overexpressing conditions (i.e. $L$. pneumophila cells without the overexpression vector and cells with this vector but without IPTG induction). However, induction of Lpa expression by $50 \mu \mathrm{M}$ IPTG revealed the plasmin-specific conversion of the chromogenic substrate S-2251. For E. coli BL21*/pMMBNlpa, plasminogen activation could be observed with and without induction of expression with $50 \mu \mathrm{M}$ IPTG. E. coli BL21* was, as expected, not able to convert Plg into plasmin. In the absence of Plg, no cleavage of S-2251 was observed, confirming the specific plasminogen activation capacity of Lpa.

Although L. pneumophila wild-type cells showed no detectable activation of plasminogen (Fig. 4a), plasminogen activation activity by an L. pneumophila wild-type outermembrane preparation, as shown in Fig. 4(c), starts to increase from about $10 \mathrm{~h}$ of incubation, corresponding to the large amount of Lpa protein found in the outer membrane following immunoblotting and detection with Lpa-specific antibodies (Fig. 3). As expected, no activity was detected in the inner-membrane protein fraction of $L$. pneumophila wild-type (results not shown), which confirms the outer-membrane localization of Lpa. The outermembrane protein fraction of L. pneumophila $\Delta l p a$ has lost the ability to activate plasminogen. Providing the lpa gene in trans on a plasmid complements this defect as can be clearly seen in Fig. 4(c). Maximum plasminogen activation by the outer-membrane fraction of the complemented strain upon induction with $50 \mu \mathrm{M}$ IPTG is reached within $5-7 \mathrm{~h}$ incubation. Also, the outer-membrane protein fraction of the lpa-overexpressing strain L. pneumophila/pMMBNlpa showed maximum activity after 5-7 h upon induction with $50 \mu \mathrm{M}$ IPTG. 
(a)

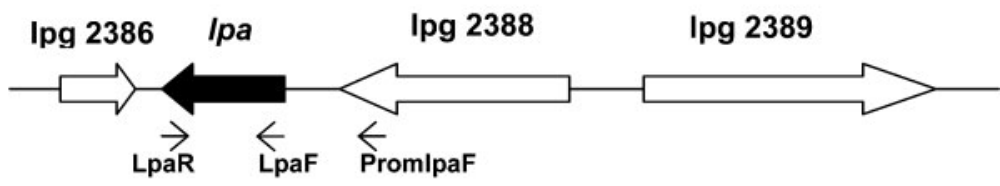

Fig. 2. Gene organization and transcriptional analysis of the Ipa region of $L$. pneumophila. (a) Schematic representation of the gene region of Ipa. (b) Agarose gel electrophoresis of RTPCR products with the indicated primer pairs. Template RNA was isolated from L. pneumophila grown to exponential (lanes 2 and 3 ) and stationary (lanes 4, 5 and 8) phase and from $L$. pneumophila grown intracellularly in $A$. castellanii (lanes 6 and 7). Control reactions were performed without reverse transcriptase to confirm the absence of DNA contamination (lanes 3, 5 and 7). Smart ladder in lane 1.

\section{Influence of O-antigen on Lpa activity}

The fact that Lpa activity of the L. pneumophila cells did not exceed background levels is remarkable, as transcriptional analysis revealed lpa expression during both the exponential and stationary growth phase (Fig. 2b). A Western blot experiment of total L. pneumophila using Lpa-specific antibodies showed expression as well, albeit at a very low level (Fig. 3). L. pneumophila might need specific conditions for an increased production of Lpa such as intracellular growth in protozoa or macrophages, as has been shown for the production of PgtE in S. enterica (Lähteenmäki et al., 2005). As well as the possibility that plasminogen activator activity did not exceed background levels due to a low in vitro expression level, it is possible that the protein is not fully active under the laboratory conditions used and that it has specific requirements with respect to, for example, the lipopolysaccharide (LPS) structure or composition. Kramer et al. (2002) showed that reconstitution of an enzymically active, purified OmpT was only achieved after addition of LPS. LPS was shown to be required for obtaining a native conformation of the protein by inducing subtle conformational changes in the surface-exposed loops. Otherwise, the L. pneumophila Lpa protein might be sterically hindered by long $\mathrm{O}-$ polysaccharides present in smooth-type LPS, as was shown

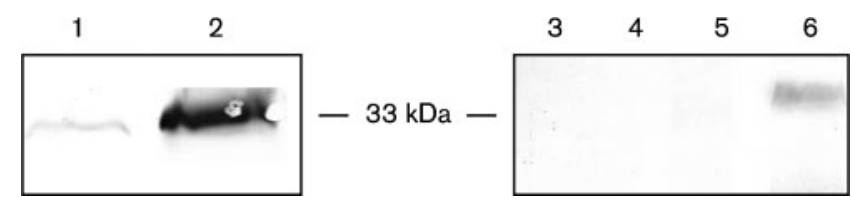

Fig. 3. Cellular localization of the L. pneumophila Lpa protein. SDS-PAGE gel electrophoresis and immunoblotting with anti-Lpa antibodies: 1 , total cell lysate $L$. pneumophila; 2 , total cell lysate $L$. pneumophila/pMMBNIpa (induced with $50 \mu \mathrm{M}$ IPTG); 3, extracellular fraction $L$. pneumophila; 4 , soluble protein fraction $L$. pneumophila; 5 , inner-membrane protein fraction L. pneumophila; 6 , outer-membrane protein fraction $L$. pneumophila. for PgtE of S. enterica (Kukkonen et al., 2004). To get an idea about the influence of the length of the O-polysaccharides on the activity of Lpa, plasminogen activation was studied in a modified LPS environment. To simulate a rough Legionella LPS background, an LPS mutant from L. pneumophila serogroup 1 strain Corby (Lück et al., 2001) was used. This LPS mutant has lost reactivity with monoclonal antibody $3 / 1$ and fails to produce high-molecular-mass long-chain $\mathrm{O}$ polysaccharides. Whereas the wild-type L. pneumophila Corby strain produces long-chain O-polysaccharides (40-75 repeating units), the LPS mutant strain produces $\mathrm{O}$ polysaccharides of highly reduced chain length of 7-15 repeating units (Lück et al., 2001). Plasminogen activation by these two strains was monitored as a function of time (Fig. 5). Although the plasminogen activation is lower compared to that of the induced L. pneumophila and E. coli Lpa-overexpressing strain, a clear difference in activity can be seen between the wild-type Corby strain and the isogenic LPS mutant strain. The LPS mutant strain shows a more than threefold increase in the plasminogen activation capacity. This experiment indicates that, like OmpT of $E$. coli, Pla of $Y$. pestis and PgtE of S. enterica, Lpa of $L$. pneumophila might also be sterically hindered by $\mathrm{O}$-antigen repeats in smooth LPS.

\section{Amoebae plate test}

Most omptins have a clear role in virulence. To analyse the possible role of Lpa in the virulence, L. pneumophila wildtype, the lpa mutant strain and the complemented strain were grown on BCYE agar plates in the presence or, as a control, in the absence of $A$. castellanii in the amoebae plate test (Albers et al., 2005). As can be seen in Fig. 6, wild-type L. pneumophila was able to grow in the presence of amoebae up to a dilution of $10^{-6}$. The lpa mutant strain formed robust colonies until a dilution of $10^{-2}$, but failed to grow from dilution $10^{-6}$, showing a partial growth defect in the presence of A. castellanii. Supplying lpa on a plasmid restored growth to the wild-type level. In the absence of $A$. castellanii all strains grew equally well. 

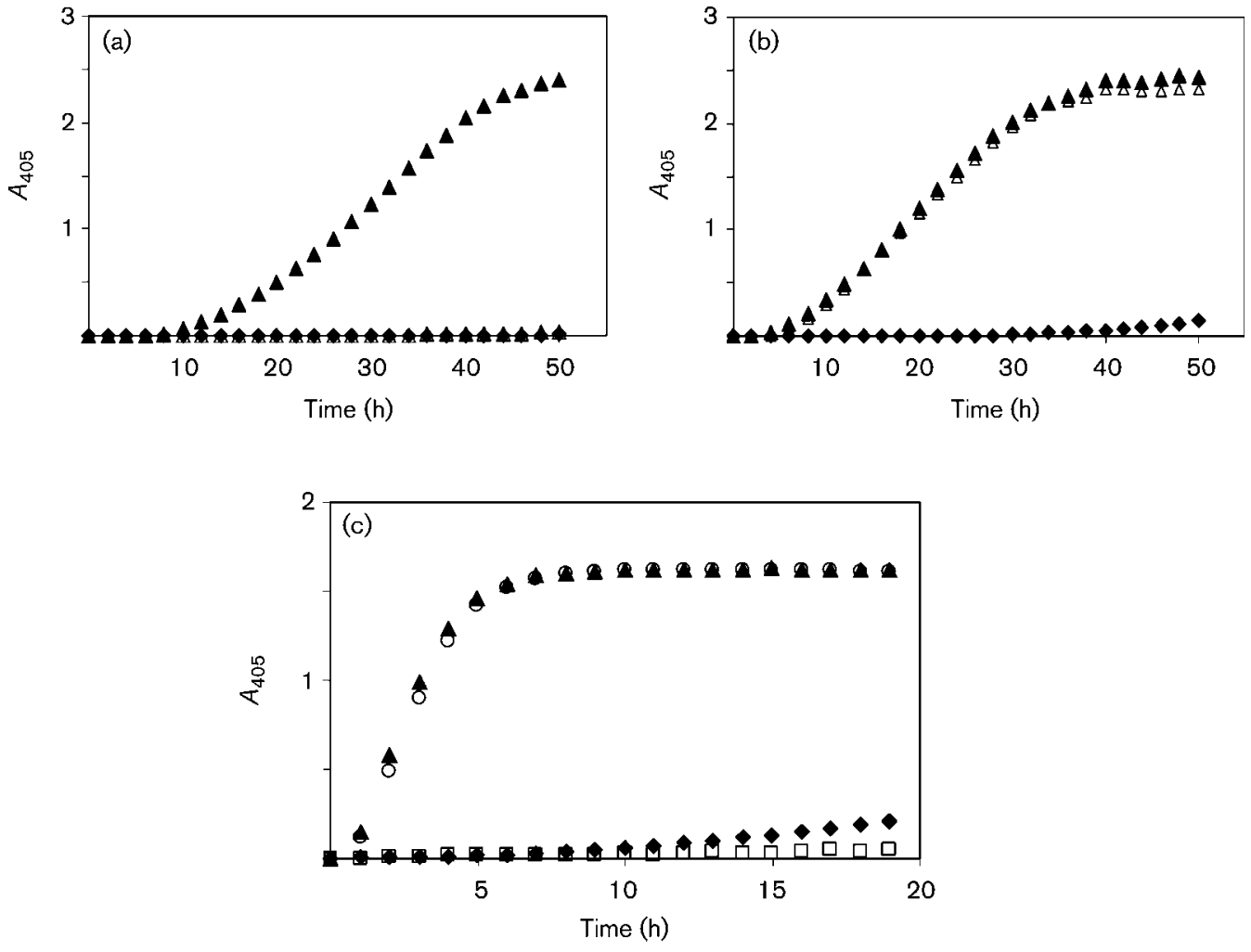

Fig. 4. Plasminogen activation as measured by the conversion of S-2251 in the presence of Glu-Plg by: (a) L. pneumophila

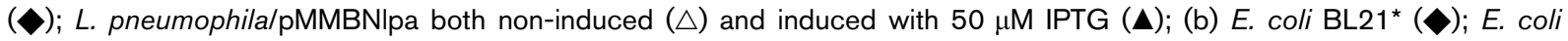
$\mathrm{BL} 21^{*} / \mathrm{pMMBN}$ pa both non-induced $(\triangle)$ and induced with $50 \mu \mathrm{M}$ IPTG $(\mathbf{\Delta})$; (c) outer-membrane fractions of $L$. pneumophila $(\diamond)$, L. pneumophila $\Delta / p a(\square)$, L. pneumophila $\Delta /$ pa/pMMBNIpa $(50 \mu \mathrm{M}$ IPTG) $(\bigcirc)$ and L. pneumophila/pMMBNIpa $(50 \mu \mathrm{M}$ IPTG) $(\boldsymbol{\Delta})$. Conversion of the substrate is given as a function of time.

\section{Conclusion}

An important prerequisite in the pathogenesis of various invasive bacterial infections is the ability of the infecting micro-organism to adhere to host tissues. Subsequent degradation of the epithelial barrier and basement membranes depends on bacterial proteases and renders penetration of the bacteria through the basement membrane and the extracellular matrix possible. For some pathogenic bacteria such as streptococci and certain Enterobacteriaceae, plasminogen activation has been found to play a key role in activating a proteolytic cascade that leads to damage of the tissue barriers.

With respect to the pathogenesis of $L$. pneumophila, research has focused almost exclusively on the interaction of the bacteria with the macrophage host. Nevertheless, besides a possible role for Lpa in the adhesion to the host cell and in intracellular replication, one might hypothesize a role in later stages of infection as well. These include penetration of the alveolar epithelial barrier and basement membrane and dissemination of the bacteria in the lung tissue, causing alveolar damage and bacterial spread. Very recently a new peptidyl prolyl cis/trans isomerase (PPIase)-dependent mechanism for bacterial tissue invasion as well as a model for alveolar damage and bacterial spread was proposed (Wagner et al., 2007). The virulence protein Mip binds collagen and renders the extracellular matrix protease sensitive in a PPIase-dependent way. An as yet not further defined serine protease

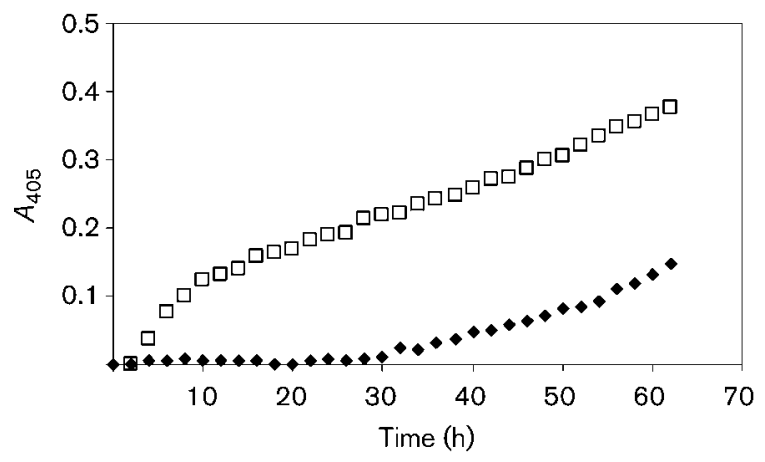

Fig. 5. Plasminogen activation as a function of time as measured by the conversion of S-2251 in the presence of Glu-Plg by $L$. pneumophila Corby WT $(\boldsymbol{)})$ and L. pneumophila Corby LPS mutant ( $\square)$. 


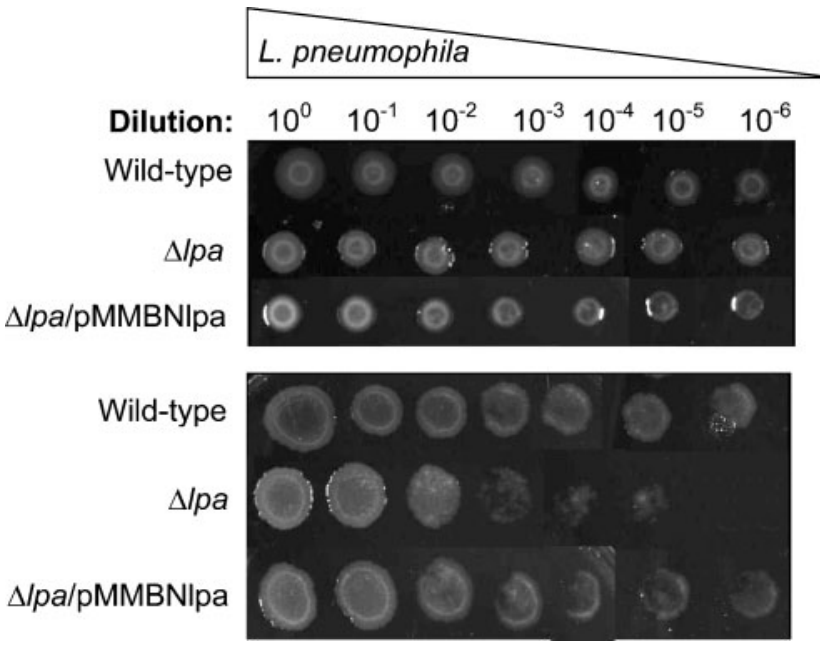

Fig. 6. Growth of wild-type L. pneumophila, the Ipa mutant strain L. pneumophila $\Delta /$ pa and the complemented strain L. pneumophila

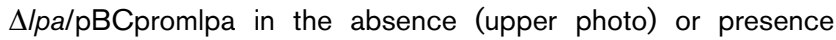
(lower photo) of $A$. castellanii in the amoebae plate test.

would contribute to the perforation of the basal membrane allowing bacterial transmigration. We hypothesize that Lpa might fulfil the role of this additional protease. We report here, to our knowledge for the first time, that $L$. pneumophila produces a protein with the capacity to convert plasminogen to plasmin. Whether this protein is important for the virulence of L. pneumophila will be the issue of further research.

\section{ACKNOWLEDGEMENTS}

L. Vranckx is a research fellow of the Institute for the Promotion of Innovation through Science and Technology in Flanders (IWTVlaanderen). This work is supported by the K. U. Leuven Onderzoeksfonds (OT project 05/62). DnaK, LepB and MOMP antibodies were a kind gift of Dr P. Mazodier, Dr G. von Heijne and Dr P. Hoffman, respectively. The wild-type and LPS mutant strain of L. pneumophila serogroup 1 strain Corby were kindly provided by $\mathrm{Dr}$ C. Lück.

\section{REFERENCES}

Albers, U., Reus, K., Shuman, H. A. \& Hilbi, H. (2005). The amoebae plate test implicates a paralogue of $l p x B$ in the interaction of Legionella pneumophila with Acanthamoeba castellanii. Microbiology 151, 167-182.

Albert-Weissenberger, C., Cazalet, C. \& Buchrieser, C. (2007). Legionella pneumophila - a human pathogen that co-evolved with fresh water protozoa. Cell Mol Life Sci 64, 432-448.

Brüggemann, H., Hagman, A., Jules, M., Sismeiro, O., Dillies, M.-A., Gouyette, C., Kunst, F., Steinert, M., Heuner, K. \& other authors (2006). Virulence strategies for infecting phagocytes deduced from the in vivo transcriptional program of Legionella pneumophila. Cell Microbiol 8, 1228-1240.
De Buck, E., Lebeau, I., Maes, L., Geukens, N., Meyen, E., Van Mellaert, L., Anné, J. \& Lammertyn, E. (2004). A putative twinarginine translocation pathway in Legionella pneumophila. Biochem Biophys Res Commun 317, 654-661.

De Buck, E., Maes, L., Meyen, E., Van Mellaert, L., Geukens, N., Anné, J. \& Lammertyn, E. (2005). Legionella pneumophila Philadelphia-1 tatB and tatC affect intracellular replication and biofilm formation. Biochem Biophys Res Commun 331, 1413-1420.

De Buck, E., Vranckx, L., Meyen, E., Maes, L., Vandersmissen, L., Anné, J. \& Lammertyn, E. (2007). The twin-arginine translocation pathway is necessary for correct membrane insertion of the Rieske Fe/ S protein in Legionella pneumophila. FEBS Lett 581, 259-264.

Edelstein, P. H. (1981). Improved semiselective medium for isolation of Legionella pneumophila from contaminated clinical and environmental specimens. J Clin Microbiol 14, 298-303.

Hilbi, H., Weber, S. S., Ragaz, C., Nyfeler, Y. \& Urwyler, S. (2007). Environmental predators as models for bacterial pathogenesis. Environ Microbiol 9, 563-575.

Kramer, R. A., Zandwijken, D., Egmond, M. R. \& Dekker, N. (2000). In vitro folding, purification and characterization of Escherichia coli outer membrane protease OmpT. Eur J Biochem 267, 885-893.

Kramer, R. A., Vandeputte-Rutten, L., de Roon, G. J., Gros, P., Dekker, N. \& Egmond, M. R. (2001). Identification of essential acidic residues of outer membrane protease OmpT supports a novel active site. FEBS Lett 505, 426-430.

Kramer, R. A., Brandenburg, K., Vandeputte-Rutten, L., Werkhoven, M., Gros, P., Dekker, N. \& Egmond, M. R. (2002). Lipopolysaccharide regions involved in the activation of Escherichia coli outer membrane protease OmpT. Eur J Biochem 269, 1746-1752.

Kukkonen, M. \& Korhonen, T. K. (2004). The omptin family of enterobacterial surface proteases/adhesins: from housekeeping in Escherichia coli to systemic spread of Yersinia pestis. Int J Med Microbiol 294, 7-14.

Kukkonen, M., Lähteenmäki, K., Suomalainen, M., Kalkkinen, N., Emödy, L., Läng, H. \& Korhonen, T. K. (2001). Protein regions important for plasminogen activation and inactivation of $\alpha_{2^{-}}$ antiplasmin in the surface protease Pla of Yersinia pestis. Mol Microbiol 40, 1097-1111.

Kukkonen, M., Suomalainen, M., Kyllönen, P., Lähteenmäki, K., Lång, H., Virkola, R., Helander, I. M., Holst, O. \& Korhonen, T. K. (2004). Lack of O-antigen is essential for plasminogen activation by Yersinia pestis and Salmonella enterica. Mol Microbiol 51, 215-225.

Lähteenmäki, K., Kyllönen, P., Partanen, L. \& Korhonen, T. K. (2005). Antiprotease inactivation by Salmonella enterica released from infected macrophages. Cell Microbiol 7, 529-538.

Lin, J., Huang, S. \& Zhang, Q. (2002). Outer membrane proteins: key players for bacterial adaptation in host niches. Microbes Infect $\mathbf{4}$, 325-331.

Lück, P. C., Freier, T., Steudel, C., Knirel, Y. A., Lüneberg, E., Zähringer, U. \& Helbig, J. H. (2001). A point mutation in the active site of Legionella pneumophila O-acetyltransferase results in modified lipopolysaccharide but does not influence virulence. Int $\mathrm{J} \mathrm{Med}$ Microbiol 291, 345-352.

Miller, J. H. (1972). Experiments in Molecular Biology. Cold Spring Harbor, NY: Cold Spring Harbor Laboratory.

Molmeret, M., Horn, M., Wagner, M., Santic, M. \& Abu Kwaik, Y. (2005). Amoebae as training ground for intracellular bacterial pathogens. Appl Environ Microbiol 71, 20-28.

Molofsky, A. B. \& Swanson, M. S. (2004). Differentiate to thrive lessons from the Legionella pneumophila life cycle. Mol Microbiol 53, 29-40. 
Morales, V. M., Backman, A. \& Bagdasarian, M. (1991). A series of wide-host-range low-copy-number vectors that allow direct screening for recombinants. Gene 97, 39-47.

Sambrook, J., Fritsch, E. F. \& Maniatis, T. (1989). Molecular Cloning: a Laboratory Manual, 2nd edn. Cold Spring Harbor, NY: Cold Spring Harbor Laboratory.

Sodeinde, O. A., Subrahmanyam, Y. V. B. K., Stark, K., Quan, T., Bao, Y. \& Goguen, J. D. (1992). A surface protease and the invasive character of plague. Science 258, 1004-1007.

Studier, F. W. \& Moffatt, B. A. (1986). Use of bacteriophage T7 RNA polymerase to direct selective high-level expression of cloned genes. J Mol Biol 189, 113-130.
Vandeputte-Rutten, L., Kramer, R. A., Kroon, J., Dekker, N., Egmond, M. R. \& Gros, P. (2001). Crystal structure of the outer membrane protease OmpT from Escherichia coli suggests a novel catalytic site. EMBO J 20, 5033-5039.

Wagner, C., Khan, A. S., Kamphausen, T., Schmausser, B., Ünal, C., Lorenz, U., Fischer, G., Hacker, J. \& Steinert, M. (2007). Collagen binding protein Mip enables Legionella pneumophila to transmigrate through a barrier of NCI-H292 lung epithelial cells and extracellular matrix. Cell Microbiol 9, 450-462.

Edited by: J. Tommassen 